drugs, if effective, would confer on the wealthy. The authors' acknowledgment of existing disturbing inequities does not mean that we should add more.

A responsible position would be to call for a moratorium on the use of enhancers until enforceable policies to minimize socioeconomic disparities are in place, research into the use and impact of these drugs is completed, information on risks and benefits is broadly disseminated - and physicians, educators and regulators have articulated professional normative positions.

Anjan Chatterjee Department of Neurology and Center for Cognitive Neuroscience, University of Pennsylvania, Philadelphia, Pennsylvania 19104, USA e-mail:anjan@mail.med.upenn.edu

\section{Recall of learned information may rely on taking drug again}

SIR - Henry Greely and colleagues identify critical areas of public discussion about perceptions and use of drugs that are alleged or expected to improve cognition.

Stimulants and other drugs proposed as potential cognitive enhancers are known to create profound state dependence, a phenomenon in which information or associations learned while 'under the influence' of a drug will later be remembered or used only when the learner has again taken the drug. Thus, individuals who use amphetamines to improve their learning of new information may indeed learn slightly faster or with less effort than those who do not use such drugs. Later, however, they may not remember or use the learned information unless they take amphetamines or related drugs again.

How and when state dependence occurs has been studied extensively in humans and other animals, in well-controlled learning tasks in laboratory settings. State dependency of cognitive enhancers would dramatically influence drug use, the permanence of learning and the ability to use information in new conditions. Indeed, state dependence can complicate clinical use of pharmacotherapies for such disorders as anxiety and attention-deficit hyperactivity disorder.

Greely and colleagues note critical research and policy questions that societies must consider to shape expectations about putative cognitive enhancers. We urge that the discussion includes the known and profound state-dependent effects of these agents.

Alice M. Young Department of Pharmacology and Neuroscience, Texas Tech University Health Sciences Center, 36014th Street, STOP 6592, Lubbock, Texas 79430, USA e-mail: alice.young@ttuhsc.edu Francis C. Colpaert Institut de Recherche Pierre Fabre, 3, rue des Satellites, BP 94244, 31432 Toulouse Cedex 4, France

\section{Patterns of drug use have varied throughout history}

SIR - Current issues are assessed by Henry Greely and colleagues, but their Commentary contains a bias consistent with current Western culture. The use of these drugs is probably older than recorded history. They have often been used regardless of social convention or rules - and have had impacts on societies.

In the 1960s, 'mind-altering drugs' had a different connotation from the present, exemplified by Timothy Leary's "Turn on, tune in and drop out" philosophy. Drugs such as marijuana and LSD were said to enhance individual thinking and creativity, but research both on the mental effects of these drugs and on their use has decreased. Since then, the use of mind-altering drugs has shifted towards enhancing performance, usually with amphetamine analogues. It seems that these are becoming acceptable when used for the goal of efficient production in standardized ways.

Don Burnap 11 Oakland Street, Rapid City, South Dakota 57701, USA

The five preceding letters are $a$ selection of many comments on this Commentary that were submitted to Correspondence. Many other reactions from readers have been posted in a long and lively online discussion forum at Nature Network, including the following anonymous contributions.

\section{Careful use helps me do better research, and society benefits}

SIR - I commend the authors of this Commentary on what I think is a fair and insightful piece. I suspect many of the negative commenters are guilty of default outrage without careful thought.

I find that my own occasional, metered use of these drugs can make astounding increases in my ability as a researcher - which results in tangible benefit to society. It's not a competition, I'm not taking an exam. I'm doing research; research that I hope may one day improve the lives of many. I exercise, sleep, eat well and I drink coffee. Yet sometimes that significant extra boost allows me to spend 12 hours successfully working through mathematics that for weeks I was previously unequal to solving. Why is this the act of a social criminal?

Current attitudes towards such drugs seem to be that they are good if you need them to become equal, but wrong if you want to become more than equal. Can we really be so quick to condemn a striving to better ourselves? Should we tell brighter students to hold back to the median? I think not. This issue is not black and white; it requires the careful grey-scale considerations the Commentary authors recommend.

It is true that many physicians and biomedical researchers have placed themselves in quite a tangled conflict-of-interest web, and that this compromise can be dangerous.

Some of the authors of this Commentary may have some conflict of interest, which thankfully Nature requires authors to disclose. However, that possible conflict of interest does not release you, the reader, from your obligation to rationally and carefully consider their argument. They are not wrong just because some of them consult for pharmaceutical companies.

\section{Enhancement means a broader role for physicians}

SIR - This Commentary raises a wider issue of perceived responsibility. The traditional role of physician as healer does not sit with the category of enhancement. Plastic surgery serves as a good analogy here, with views remaining varied on the ultimate responsibility. But with planned, novel, pharmacological intervention, physicians must surely be party to the cause, and thus a broadening of their role would be necessary.

Ultimately, the call for risk-benefit research and a fuller understanding of mechanisms is therefore welcome, but the real beneficiaries, at least in the short term, are likely to be dominated by the genuinely needy - neurological and psychiatric patients. These groups have had their needs for cognitive enhancement unmet for far too long.

Further discussion of the Commentary and these Correspondence contributions is welcome at Nature Network. Please visit http://tinyurl. com/6nyu29 to have your say. 